\title{
Ropivacaine Induced Brachial Plexus Neurotoxicity- A Case Report
}

\author{
Shailesh Murty ${ }^{1}$, Kanishk Murty ${ }^{2}$
}

\section{Abstract}

Background: Neurotoxicity of the nerves has been reported to be related to the concentration or volume of local anaesthetic used.

We report a case wherein the volume and concentration of local anaesthetic used was well within the safe limits. Despite the safe precautions taken, the patient developed a profound motor and sensory block which took nearly 20 weeks to resolve.

Case presentation: A 72-year-old female patient scheduled for a total shoulder arthroplasty received a single shot interscalene block under ultrasound guidance. A total volume of $10 \mathrm{mls}$ of ropivacaine $0.375 \%$ was injected under direct ultrasound guidance. The patient experienced no pain on injection and neither was there any paraesthesia on needle placement. We encountered no high pressures during the injection. The patient had a profound block which lasted for over 20 weeks. The patient was followed up at regular intervals which showed a slow but complete recovery pattern.

Conclusion: Local anaesthetics are known to cause neurotoxicity. This case highlights the need to be mindful about potential sequelae to nerve blocks despite using a lower concentration and volume of local anaesthetic.

Keywords: Interscalene block; Neurotoxicity; Ropivacaine.

\section{Introduction}

We report a case of prolonged nerve damage following a single shot ultrasound guided Interscalene brachial plexus nerve block. Although the risk of neurological complications from peripheral nerve blocks is reported to be $0.02 \%-0.5 \%$ [ 1 ], on thorough neurological examination this value was recorded to be closer to $0.04 \%$ [2]. Nerve damage can be due to direct needle trauma or due to local anaesthetic induced neurotoxicity. Intrafascicular blocks can potentially lead to mechanical injury as well as exposing the nerves to higher concentrations of local anaesthetics leading to neurotoxicity.

Patient Information And Clinical Findings A 72-year-old female patient presented for a right total shoulder arthroplasty. She had a history of paroxysmal atrial fibrillation and well controlled hypertension. She weighed 52 kilograms and was $162 \mathrm{cms}$ tall. The patient was consented for a single shot ultrasound guided interscalene nerve block along with a general anaesthetic. The procedure was carried out with the patient awake under sedation and an in-plane approach was used and the needle tip advanced in between the nerve trunks. The injections were carried out at multiple levels. The first injection was done below the lowest nerve trunk, the second was done between the lower and middle trunks and the final was done above the top most nerve trunk [3]. A total volume of $10 \mathrm{mls}$ of $0.375 \%$ plain ropivacaine was injected after careful aspiration after each needle placement. The injections were done by the anaesthetist performing the block and no high pressures were encountered during the multiple injections. Good shoulder anaesthesia was achieved within fifteen minutes. The intraoperative course was uneventful. The patient had a profound nerve block the following morning with near total motor loss and complete sensory loss. An ultrasound of the neck was done which showed swollen nerve trunks which was attributed to possible neurotoxicity. On day two, a decision was made to do a $\mathrm{T} 2$ weighted MRI scan of the neck and shoulder. The MRI scan showed an enlarged anterior scalene muscle with increased T2 signalling. Furthermore, the MRI revealed fluid tracking into the scalene triangle and more laterally extending $4 \mathrm{cms}$ in the craniocaudal dimension. Apart from the scalene triangle, the brachial plexus was normal. The patient was reassured about the prolonged block and was discharged with fortnightly consults, looking for neurological improvement [4]. There was slow but complete recovery of motor and sensory function which took over 20 weeks to completely resolve. The sensory recovery was quicker than the motor.

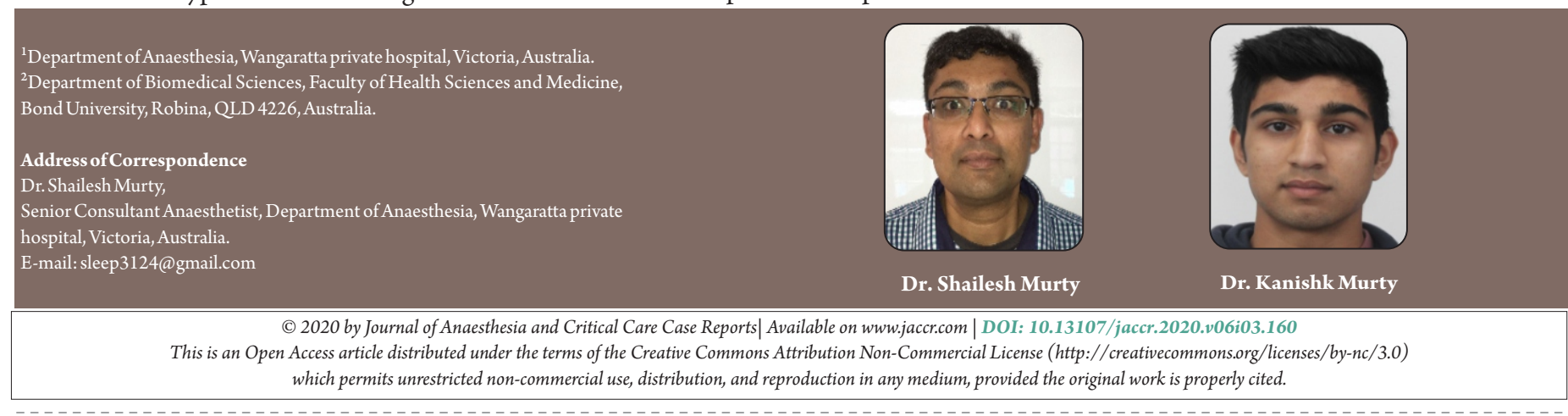

22 | Journal of Anaesthesia and Critical Care Case Reports | Volume 6 | Issue 3 | September-December 2020 | Page 22-24 


\section{Figure 1: Algorithm for management of nerve injury associated with regional anaesthesia}

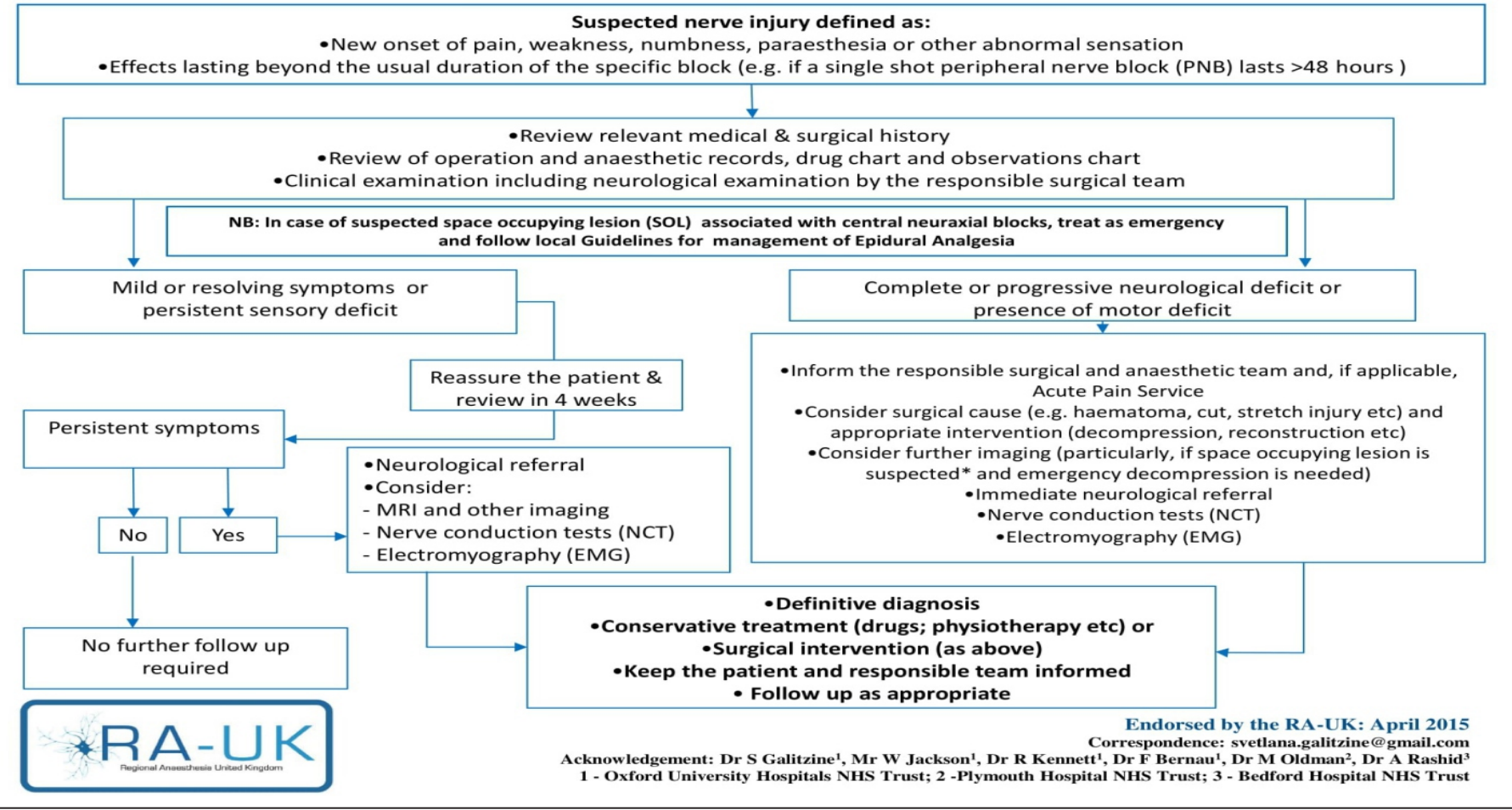

Timeline

Ultrasound guided Interscalene block (week0)

Profound motor and sensory loss (day 1 post operation)

USG scan showed swollen nerve trunks (day 2 post operation)

MRI scan showed fluid around the nerve trunks (day 2 post operation)

Follow up done over the next 20 weeks showed slow but complete recovery

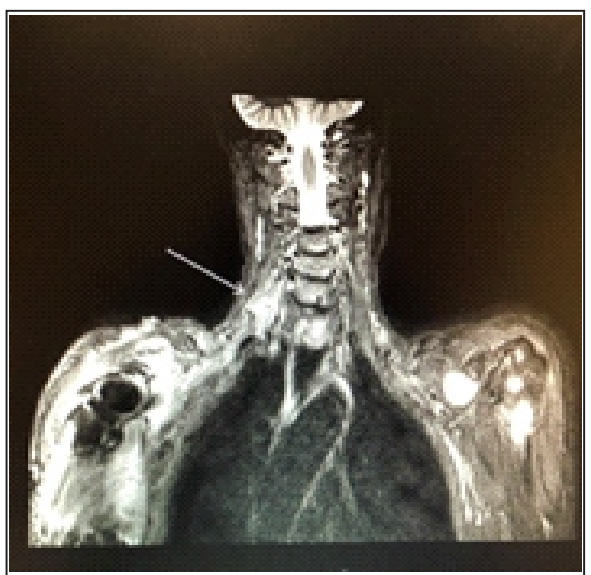

Figure 2: T2 weighted coronal section of MRI showing enlarged nerve trunks

\section{Investigations}

An ultrasound of the neck and an MRI were done on day 2 post procedure. The ultrasound showed swollen nerve trunks which were attributed secondary to local anaesthetic toxicity. The MRI showed fluid present around the nerve trunks.

Therapeutic Intervention

The patient was advised intensive physiotherapy post procedure. A high dose vitamin B12 oral therapy was also instituted 3 weeks post procedure [4].

\section{Follow-Up and Outcome}

The patient was followed up fortnightly and

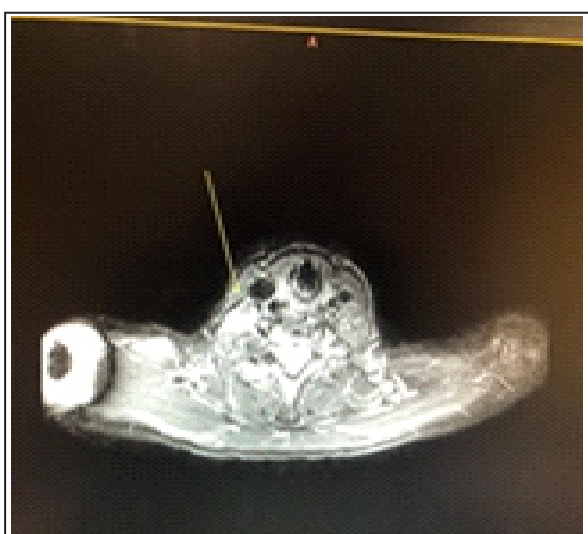

Figure 3: T2 weighted transverse section of MRI showingnenlarged nerve trunks and fluid in the interscalene space made a complete recovery within 20 weeks.

\section{Discussion}

Amongst peripheral nerve blocks, there is a higher incidence of nerve injury with brachial plexus blocks [6]. With the presence of a near total motor and compete sensory loss of function, it was deliberated if this was an anaesthetic or surgical cause. There was no intraoperative traction and the head was in a fixed position throughout the case. The ultrasound done on the following day did show swollen nerve trunks and we were fairly convinced this was because of ropivacaine induced neurotoxicity. The T2 weighted MRI scan confirmed fluid in the scalene triangle

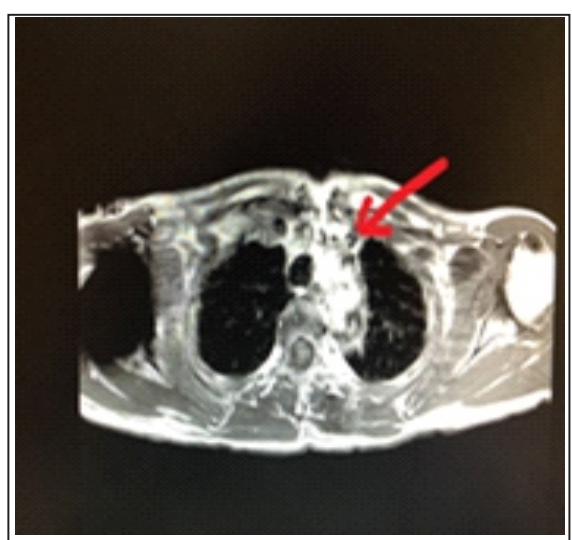

Figure 4: T2 weighted transverse section of MRI showing enlarged nerve trunks and fluid in the interscalene space

23 |Journal of Anaesthesia and Critical Care Case Reports | Volume 6 | Issue 3 | September-December 2020| Page 22-24 
with extension in the craniocaudal dimension on day two post operatively. A nerve conduction study was deliberated but it would take up to 3 weeks for signs of Wallerian degeneration to appear, by which time the patient showed signs of improvement and recovery. We had to work out if the cause was mechanical, chemical or a combination of the two. There was no paraesthesia or pain on needle placement which made us fairly certain that direct needle trauma was not plausible. The concentration oflocal anaesthetic used was quite low and the dosage given was well within the normal range. A total of 37.5 milligrams of Ropivacaine was used in a patient weighing 52 kilograms. There was no high pressure encountered during the injections as these were carried out by the anaesthetist performing the block. Ropivacaine has a greater safety profile, however, there have been reports of neurotoxicity at larger concentrations or volumes. We have never encountered a case of neurotoxicity from ropivacaine at such small doses [6]. Given the recovery it was unlikely this was an intrafascicular injection which would normally lead to axonal degeneration and permanent nerve damage. It could be quite likely that this was a result of an intraneural needle placement as there are documented case reports of neural injury that have taken up to 6 months to resolve [7]. The brachial plexus at the level of the trunks is nearly all neural tissue, a needle entering the nerve here is more likely to encounter a fascicle on its trajectory that may contribute to the disproportionately higher rate of postoperative neuropathy [8]. The motor and sensory recovery started from the shoulder muscles and dermatomes and proceeded distally. The hand was the last to recover. We are aware that most of the local anaesthetic injected diffuses out of the brachial plexus sheath fairly quickly and as such we were quite perplexed by the presence of fluid within the scalene triangle. The ulnar distribution recovered before the radial and medial distributions. As the medial cord is simply a continuation of the anterior division of the lower trunk (C8, T1), we hypothesised that it could have been residual local anaesthetic within the interscalene sheath which led to prolonged exposure of the nerve trunks to the local anaesthetic. The presence of a hematoma would have had a hyper to hetero intense image which was absent [9]. Furthermore, the mass effect from a hematoma would be a completely different presentation.

\section{Conclusion}

Although all local anaesthetics are known to be neurotoxic, ropivacaine has a greater safety profile. The exact mechanism of nerve damage could not be identified. We were fairly certain that mechanical trauma could not be a causative factor. The patient had no metabolic medical conditions that could have precluded her to have a higher incidence of nerve damage. The presence of fluid within the interscalene sheath on day 2 made us suspect residual local anaesthetic that had perhaps not diffused out or absorbed. The presence of persistent local anaesthetic around the nerve trunks could have led to a longer exposure of the trunks to the local anaesthetic resulting in nerve damage, which in itself would be highly unusual. We were fairly certain that the cause could be due to local nerve neurotoxicity or from an intraneural needle placement.

\section{References}

1. Brull R., McCartney C.J., Chan V.W., El-Beheiry H. Neurological complications after regional anesthesia: Contemporary estimates of risk. Anesth. Analg. 2007; 104:965-974

2. Barrington M.J., Watts S.A., Gledhill S.R., Thomas R.D., Said S.A., Snyder G.L., Tay V.S., Jamrozik K. Preliminary results of the australasian regional anaesthesia collaboration: A prospective audit of more than 7000 peripheral nerve and plexus blocks for neurologic and other complications. Reg. Anesth. Pain Med. 2009; 34:534-541

3. Pathophysiology and Etiology of Nerve Injury Following Peripheral Nerve Blockade. Brull R, Hadzic A, Reina MA, Barrington MJ Reg Anesth Pain Med. 2015 Sep-Oct; 40(5):479-90.

4. Ehmedah A, Nedeljkovic P, Dacic S, et al. Vitamin B Complex Treatment Attenuates Local Inflammation after Peripheral Nerve Injury. Molecules. 2019;24(24):4615. Published 2019Dec 17. doi:10.3390/molecules 24244615

5. Oflaherty, Doireann \& McCartney, C.J.L. \& Ng, Su. (2018). Nerve injury after peripheral nerve blockade - current understanding and guidelines. BJA Education. 18.10.1016/j.bjae.2018.09.004. DOI: 10.1016/j.bjae.2018.09.004.

6. Evaluation of brachial plexus anesthesia for upper extremity surgery. Urban $M K$ Urquhart B. Reg Anesth. 1994May-Jun; 19(3):175-82

7. Robards C, Hadzic A, Somasundaram L, Iwata T, Gadsden J, Xu D, Sala-Blanch X: Intraneural injection with low-current stimulation during popliteal sciatic nerve block. Anesth. Analg 2009; 109:673-7

8. Liu SS, Zays VM, Gordon MA, et al. A prospective, randomized, controlled trial comparing ultrasound versus nerve stimulator guidance for interscalene block for ambulatory shoulder surgery for postoperative neurological symptoms. Anesth Analg. 2009; 109(1): 265-271.

9. Chhabra A, Ahlawat S, Belzberg A, Andreseik G. Peripheral nerve injury grading simplified on MR neurography: As referenced to Seddon and Sunderland classifications. Indian J Radiol Imaging. 2014;24(3):217-224. doi:10.4103/0971-3026.137025

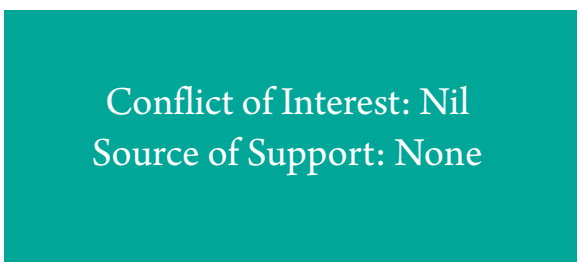

How to Cite this Article

Murty S, Murty K | Ropivacaine Induced Brachial Plexus Neurotoxicity- A Case Report | Journal of Anaesthesia and Critical Care Case Reports | September-December 2020; 6(3): 22-24. 